\title{
Management of bleeding problems with hormone replacement therapy
}

\author{
Tim Hillard, DM, FRCOG, Consultant Obstetrician and Gynaecologist, Poole General Hospital, Dorset, UK
}

Correspondence: Mr Tim Hillard, Department of Obstetrics and Gynaecology, Poole General Hospital, Longfleet Road, Poole, Dorset BH15 2JB, UK. Tel: +44 (0) 1202 442511.E-mail: thillard@poole-tr.swest.nhs.uk

(Accepted $27^{\text {th }}$ July 2002)

The Journal of Family Planning and Reproductive Health Care 2002: 28(4): 182-184

\section{Introduction}

It is well established that all non-hysterectomised women taking oestrogen replacement therapy should receive additional progestogen to reduce the incidence of endometrial hyperplasia ${ }^{1-3}$ and cancer. ${ }^{4}$ Progestogens are either added cyclically on a monthly (sequential), or 3monthly basis (tricyclic) or continuously (period-free or no bleed). Alternative combinations such as bimonthly, ${ }^{5}$ 6monthly ${ }^{6}$ and intermittent where the progestogen is added for 3 days every 3 days have been tried. ${ }^{7}$ However, data on these preparations are limited, and there are no licensed products in the UK utilising these schedules. Choosing the appropriate combination is a key factor in minimising the likelihood of unscheduled bleeding with hormone replacement therapy (HRT). The introduction of period-free HRT in perimenopausal women, often in the hope that it will stop their bleeding, is probably one of the biggest causes of bleeding problems with HRT. Continuous progestogen addition should be reserved for women who are clearly postmenopausal. This is often taken to be over 54 years, as by this stage over $80 \%$ of women will be postmenopausal, but there is no reason why younger women cannot commence it if they are clearly postmenopausal.

Unscheduled bleeding on HRT is a common problem and is one of the biggest causes of dissatisfaction with HRT. With advancing age the potential for underlying pathology increases, thus the two main strategies in managing irregular bleeding on HRT are first to exclude pathology and second to correct the irregular bleeding. Bleeding problems are not only inconvenient and a cause of anxiety, they are also costly. Ettinger et al. reported that over $38 \%$ of women on sequential and $41 \%$ on period-free HRT attended his clinic with bleeding irregularities in a 12 -month period, and $12 \%$ and $20 \%$, respectively, required endometrial biopsies. ${ }^{8}$

Various types and routes of progestogens are used in HRT. The most widely used are the testosterone derivatives, norethisterone acetate (NETA) and levonorgestrel, and the C21 derivatives, medroxyprogesterone acetate (MPA) and dydrogesterone. Other progestogens that are used with HRT include trimegestone,${ }^{9}$ cyproterone acetate, ${ }^{10}$ nomegestrol ${ }^{11}$ and norgestimate. ${ }^{12}$ The third-generation levonorgestrel derivatives, desogestrel and gestodene, were being actively looked at as part of HRT combinations but since their possible association with an increased thrombosis risk in the combined pill, little progress has been made. Natural progesterone is available in the UK as either vaginal pessaries or sustained-release vaginal ge ${ }^{13}$ and can be used as an alternative for progestogen intolerant women. This should not be confused with 'natural' progesterone cream, which is available as a natural health product but has no proven efficacy on the endometrium. ${ }^{14}$. Oral micronised progesterone may also be used but is not readily available in the UK. NETA and levonorgestrel are available transdermally as part of an oestrogen combination patch. The levonorgestrel intrauterine system (IUS) is increasingly being used for the progestogen component of HRT. Whilst it appears to be effective in opposing the oestrogenic effects on the endometrium and it also solves the vexing problem of contraception in perimenopausal women, it is not currently licensed for use with HRT, and the few published studies have very small numbers. ${ }^{15}$ Further trials are ongoing.

Choosing the progestogen for a particular woman is an individual decision. Side effects are common with all progestogens and may be improved by changing the type or dose of the progestogen. Whilst there are some differences between the metabolic effects of the various progestogens, the clinical relevance of these is unclear and caution should be exercised in interpreting data on surrogate markers of disease end points such as cardiovascular disease and thrombosis. As far as cycle control is concerned there are no clear data to indicate that one progestogen is superior to another. The recommended doses for cyclical administration are well known ${ }^{16}$ and commercial preparations incorporate these. When particular difficulties arise, the progestogens can be prescribed individually as part of a tailor-made combination.

\section{Causes of unscheduled bleeding on HRT}

On sequential HRT $85 \%$ women get a regular withdrawal bleed and up to $15 \%$ have no bleeding. ${ }^{16}$ This is acceptable provided the other benefits of HRT, e.g. symptom control, are realised. Problems may occur with either the timing of the withdrawal bleed, the length or heaviness of the bleed, or with breakthrough bleeding. Depending on the age group, pregnancy should be excluded, compliance checked and pelvic examination performed unless done recently. Antibiotics, particularly the broad-spectrum ones, may interfere with hormone absorption. Synchronisation of the endogenous ovarian cycle and the exogenous hormone cycle in perimenopausal women can be difficult. Unlike the combined contraceptive, HRT does not suppress endogenous ovarian activity and thus bleeding may occur twice per month.

Quarterly bleed preparations have the advantage of only resulting in a bleed four times a year. Although potentially advantageous, breakthrough bleeding may occur in the oestrogen-only phase, and the higher doses of progestogen used at the end of the cycle may be more likely to cause side effects. Concerns exist that prolonged oestrogen stimulation may lead to higher rates of hyperplasia and carcinoma ${ }^{17}$ and such preparations should be used with caution in the long term. 
With period-free or no-bleed HRT, the underlying principle is that progestogen is continuously added to oestrogen to oppose the proliferative effects of oestrogen on the endometrium and thus prevent bleeding. In practice, the occurrence of bleeding, particularly in the first 6 months, is commonplace and in some cases may be attributable to starting the preparation too early (see above). The majority of women achieve amenorrhoea by 6 months and amenorrhoea rates can be maximised by confining the use of these preparations to truly postmenopausal women. ${ }^{18}$ Once amenorrhoea is achieved it is usually maintained. Differences do exist between products, with less initial bleeding reported with the lower dose combinations ${ }^{19}$ and Tibolone. ${ }^{20}$ Interpretation of different studies is hampered by variable reporting of bleeding episodes. ${ }^{21}$ Because bleeding is frequent in the first 3 months of treatment with this type of preparation, it is not usually considered abnormal. Women should be advised of the risk of such bleeding prior to starting treatment. Women switching from sequential to period-free HRT may achieve amenorrhoea more rapidly by having a washout period. ${ }^{22}$ In the absence of clinical signs or other risk factors, bleeding between 3 and 6 months is usually considered to be acceptable but persistent bleeding after 6 months or bleeding commencing after a spell of amenorrhoea should always be investigated. Benign pathology, such as endometrial polyps and submucous fibroids, are common, occurring in up to $40 \%$ of women. ${ }^{23}$ Other genital tract lesions and ovarian pathology need to be excluded. In the majority of cases no pathological cause for the bleeding is found and the endometrium is atrophic.

The risk of endometrial hyperplasia associated with sequential HRT over approximately 3 years is around 2.7$5 \%{ }^{3,24}$ With tricyclic HRT it may be as high as $11 \% .^{17}$ Conversely, the risk of endometrial hyperplasia with periodfree HRT is less than $1 \% .^{3,25}$ Indeed, continuous progestogen is an effective method of converting hyperplasia back to normal. ${ }^{24}$ Recent epidemiological studies have indicated that more than 5 years of sequential HRT in postmenopausal women is associated with a three-fold increase in endometrial cancer risk, ${ }^{26,27}$ whereas continuous progestogen addition was associated with a four- to five-fold reduction in cancer risk. On the basis of these results women should be advised to switch to no-bleed combinations once they are 5 or more years past menopause. In reality many such women will be on these combinations already. The same study suggested that the incidence of endometrial cancer may be lower with the testosterone-derived progestogens than the $\mathrm{C} 21$ progestogen derivatives ${ }^{27}$ but further studies are needed.

\section{The management of unwanted bleeding}

Although bleeding problems are common with HRT there are very few studies that have specifically looked at strategies to overcome them. The following recommendations are thus based on clinical experience and previous reviews on the subject ${ }^{28,29}$ but are likely to have some resonance with those regularly prescribing hormonal contraceptives. Clearly if a potential cause for unscheduled bleeding is identified then this should be corrected or treated first.

\section{Sequential HRT}

Synchronisation with any endogenous activity is necessary. The progestogen phase of HRT should coincide with the luteal phase of the cycle, which is the last 12-14 days of the cycle. Although natural cycle lengths may vary considerably, the luteal phase is consistent. Thus for a 28-day cycle progestogens should start around Day 15-17 and for a 35-day cycle they should start around Day 21. If cycle control remains difficult, a higher dose of progestogen may be necessary. The monophasic, low-dose oral contraceptive can also be used, the higher potency being strong enough to suppress endogenous activity. The levonorgestrel IUS is an alternative option, although unlicensed.

The withdrawal bleed should occur at the end of the progestogen phase. If the withdrawal bleed is heavy or prolonged or occurs too early, then increasing the dose or changing the type of progestogen may be helpful. Spotting before the withdrawal bleed may represent inadequate endometrial stromal formation and a higher oestrogen dosage could be used. If bleeding problems are persistent then referral for further investigation should be considered.

\section{Period-free HRT}

As indicated above, amenorrhoea is achieved by 6 months in the majority of women. Those furthest from the menopause appear to have the highest incidence of amenorrhoea. ${ }^{18,29}$ Persistent bleeding beyond this should be investigated. Ginsburg and Prelevic ${ }^{30}$ reported on 8 years' experience with treating over 400 women with Tibolone: $12 \%$ of these women experienced some bleeding, $4 \%$ having had recent oestrogen exposure. However, of the remaining 47 women, just under half had an underlying structural abnormality such as endometrial polyps, fibroids, endometrial hyperplasia or carcinoma.

In the absence of endometrial pathology, increasing the dose or changing the progestogen may help. Bleeding problems are less with lower dose preparations, but there are some women who seem to bleed persistently on no-bleed HRT. A small oestrogen supplement may help in some women, but if all fails then the women may be best advised to revert to sequential HRT and a regular bleed. Whilst the levonorgestrel IUS is another option, persistent spotting with this method cannot be ruled out. Endometrial resection has been suggested, ${ }^{31}$ but the justification for such a surgical procedure needs to be quite strong. The advent of newer outpatient techniques may change the balance on this issue in the future.

\section{Investigation of abnormal bleeding}

When investigation is required the methods employed will depend on local protocols and services. However, this should involve a pelvic examination and at least one of: transvaginal ultrasound (TVUS), hysteroscopy or endometrial biopsy. The accuracy of TVUS can be enhanced further by the technique of saline infusion hystosonography (SIS). Whilst hysteroscopy remains the gold standard investigation and outpatient hysteroscopy facilities are now becoming increasingly available, the use of TVUS and endometrial biopsy is an acceptable alternative. However, unlike the clinical scenario of postmenopausal bleeding, there is no agreed endometrial cut-off for women taking HRT. ${ }^{29}$ In their comprehensive review, Spencer and Whitehead ${ }^{32}$ demonstrated that whilst mean endometrial thickness (ET) for women on continuous combined HRT was only $4.5 \mathrm{~mm}$, there was a wide range of measurements $(1-17 \mathrm{~mm})$ all associated with no underlying pathology. For women on Tibolone the margin was much tighter, with all women having an ET of less than $4 \mathrm{~mm}$ (mean $3.2 \mathrm{~mm}$ ). Current data would suggest that for women on no-bleed HRT the same cut-off should be applied as for postmenopausal bleeding. For women on sequential HRT an ET above $8 \mathrm{~mm}$ should be investigated. Between 5 and $8 \mathrm{~mm}$ it may be reasonable to rescan and perform a biopsy if concern persists. $^{33}$ 


\section{Conclusions}

Progestogens are an essential component of HRT in nonhysterectomised women, but are also responsible for many of the problems associated with it. Bleeding problems are common with both sequential and period-free preparations and may result in disillusionment and early discontinuation of treatment. Choosing the correct type of preparation from the outset is one of the keys to the successful prescribing of HRT. Treatment should then be adjusted according to individual requirements and the women followed up until settled on a particular combination. Whilst there may be pressure to introduce 'no bleed' preparations early on, this may be counterproductive and these combinations should be reserved for women who are clearly postmenopausal. Equally, although a 'standard' preparation may suit the majority of women, there are a number who require more individual prescribing and those involved in establishing local formularies should be encouraged to provide a realistic range of alternatives from the huge array of preparations available. In the event of unscheduled bleeding, investigation is necessary according to local protocol. Whilst the incidence of underlying benign pathology may be quite high, the likelihood of malignancy is low and unless there is particular clinical concern these women do not need fasttrack referral which should be reserved for women with true postmenopausal bleeding.

\section{Statements on funding and competing interests}

Funding. The author has acted in an advisory capacity to several pharmaceutical companies in relation to hormone replacement therapy and has received grants for research and lectures from a number of pharmaceutical companies.

Competing interests. None identified.

\section{References}

1 Sturdee DW, Wade-Evans T, Patterson MEL, et al. Relationship between bleeding patterns, endometrial histology and oestrogen treatment in menopausal women. BMJ 1978; 1: 1575-1577.

2 Whitehead MI, Townsend PT, Pryse-Davies J, et al. Effects of oestrogen and progestins on the biochemistry and morphology of the postmenopausal endometrium. N Engl J Med 1981; 305: 1599-1605.

3 The Writing Group for the PEPITrial. Effects of hormone replacement therapy on endometrial histology in postmenopausal women: the Postmenopausal Estrogen/Progestin Interventions Trial. JAMA 1996; 275: 370-375.

4 Persson I, Adami HO, Bergvist L, et al. Risk of endometrial cancer after treatment with oestrogen alone or in conjunction with progestogens: results of a prospective study. BMJ 1989;298: 147-151.

5 DeLeo V, la Marca A, Morgante G, et al. Comparison of two HRT regimens with bimonthly and monthly progestin administration in postmenopause. Maturitas 1999; 31: 171-177.

6 Ettinger B, Pressman A, Van Gessel A. Low-dosage esterified estrogens opposed by progestin at 6-month intervals. Obstet Gynecol 2001; 98: 205-211.

7 Rozenberg S, Caubel P, Lim PC. Constant estrogen, intermittent progestogen vs continuous combined hormone replacement therapy: tolerability and effect on vasomotor symptoms. Int J Gynecol Obstet 2001; 72: 235-243.

8 Ettinger $\mathrm{B}, \mathrm{Li} \mathrm{DK}$, Klein $\mathrm{R}$. Unexpected vaginal bleeding and associated gynecological care in postmenopausal women using hormone replacement therapy: comparison of cyclic versus continuous combined schedules. Fertil Steril 1998; 69: 865-869.

9 Al-Azzawi F, Wahab M, Thompson J, et al. Acceptability and patterns of endometrial bleeding in estradiol-based HRT regimens: a comparative study of cyclical sequential combinations of trimegestone or norethisterone acetate. Climacteric 2001; 4: 343-354.

10 Koninckx PR, Lauweryns JM, Cornille FJ. Endometrial effects during hormone replacement therapy with a sequential, oestradiol valerate/cyproterone acetate preparation. Maturitas 1993; 16: 97-110.
11 Blanc B, Cravello L, Micheletti MC, et al. Continuous hormone replacement therapy for menopause combining nomegestrol acetate and gel, patch or oral estrogen: a comparison of amenorrhea rates. Clin Ther 1998; 20: 901-912.

12 Curran MP, Wagstaff AJ. Estradiol and norgestimate. A review of their combined use as hormone replacement therapy in postmenopausal women. Drugs Aging 2001; 18: 863-885.

13 Ross D, Cooper AJ, Pryse-Davies J, et al. Randomised double-blind dose ranging study of the endometrial effects of a vaginal progesterone gel in estrogen treated postmenopausal women. Am J Obstet Gynecol 1997; 177: 937-941.

14 Drugs and Therapeutics Bulletin. "Natural" progesterone creams for postmenopausal women. Drug Ther Bull 2001; 39: 10-11.

15 Suvanto-Luukkonen E, Kauppila A. The levonorgestrel intrauterine system in menopausal hormone replacement therapy: 5 year experience. Fertil Steril 1999; 72: 161-163.

16 Whitehead MI, Hillard TC, Crook D. The role and use of progestogens. Obstet Gynecol 1990; 75: 559-576.

17 Cerin A, Heldaas K, Moeller B. Adverse endometrial effects of longcycle estrogen and progestogen replacement therapy. $N$ Engl J Med 1996; 334: 668-669.

18 Archer DF, Dorin $\mathrm{MH}$, Heine W, et al. Uterine bleeding in postmenopausal women on continuous therapy with estradiol and norethindrone acetate. Obstet Gynecol 1999; 94: 323-329.

19 Stadberg E, Mattsson L-A, Uvebrant M. 17 -Estradiol and norethisterone acetate in low doses as continuous combined hormone replacement therapy. Maturitas 1996; 23: 31-39.

20 Hammar M, Christau S, Nathorst-Boos J, et al. A double blind randomised study comparing the effects of tibolone and continuous combined hormone replacement therapy in postmenopausal women with menopausal symptoms. Br J Obstet Gynaecol 1998; 105: 904-911.

21 Archer DF Pickar JH. The assessment of bleeding patterns in postmenopausal women during continuous combined hormone replacement therapy: a review of methodology and recommendations for reporting of the data. Climacteric 2002; 5: 45-59.

22 Hillard TC, Lumsden MA, Rees M, et al. Bleeding on sequential HRT and endometrial thickness predict success of transition from sequential hormone replacement therapy to continuous combined transdermal hormone replacement therapy. J Br Menopause Soc 2001; 7(Suppl. 3): 18-19.

23 Nagele F, O'Connor H, Basket TF, et al. Hysteroscopy in women with abnormal uterine bleeding on hormone replacement therapy: a comparison with postmenopausal bleeding. Fertil Steril 1996; 65: 1145-1150.

24 Sturdee DW, Ulrich LG, Barlow DH, et al. The endometrial response to sequential and continuous combined oestrogen-progestogen replacement therapy. Br J Obstet Gynecol 2001; 107: 1392-1400.

25 Archer DF, Pickar JH, Bottiglioni F. Bleeding patterns in postmenopausal women taking continuous combined or sequential regimens of conjugated estrogens with medroxy-progesterone acetate. Obstet Gynecol 1994; 83: 686-692.

26 Beresford SA, Weiss NS, Voigt LF, et al. Risk of endometrial cancer in relation to use of oestrogen combined with cyclic progestogen therapy in postmenopausal women. Lancet 1997; 349: 458-461.

27 Weiderpass E, Adami HO, Baron JA, et al. Risk of endometrial cancer following estrogen replacement with and without progestins. J Natl Cancer Inst 1999; 91: 1131-1137.

28 Spencer CP, Cooper AJ, Whitehead MI. Management of abnormal bleeding in women receiving hormone replacement therapy. BMJ 1997; 315: 37-42.

29 Hillard TC. Period free HRT. In: Sturdee D, Olah K, Keane D (eds), Yearbook of obstetrics \& gynaecology. London: RCOG Press, 2001; 98-118.

30 Ginsburg J, Prelevic GM. Cause of vaginal bleeding in postmenopausal women taking Tibolone. Maturitas 1996; 24: $107-110$.

31 Romer T. Treatment of recurrent bleeding disorders during hormone replacement therapy (HRT) by transcervical endometrial ablation. Gynecol Obstet Invest 1999; 47: 255-257.

32 Spencer CP, Whitehead MI. Endometrial assessment revisited. Br J Obstet Gynaecol 1999; 106: 623-632.

33 Melson L, Hillard TC. The management of abnormal bleeding on HRT - a proposed protocol and early audit of results. J Br Menopause Soc 2002; 4(Suppl. 2): in press. 